\title{
Fabrication Technology of and Symmetry Breaking in Superconducting Quantum Circuits
}

\author{
T. Niemczyk ${ }^{1}$, F. Deppe ${ }^{1,2}$, M. Mariantoni $i^{1,2}$, E.P. Menzel ${ }^{1}$, \\ E. Hoffmann ${ }^{1,2}$, G. Wild ${ }^{1,2}$, L. Eggenstein ${ }^{1,2}$, A. Marx ${ }^{1}$, R. \\ Gross $^{1,2}$ \\ ${ }^{1}$ Walther-Meißner-Institut, Bayerische Akademie der Wissenschaften, D-85748 \\ Garching, Germany \\ ${ }^{2}$ Physik-Department, Technische Universität München, D-85748 Garching, \\ Germany \\ E-mail: Rudolf.Gross@wmi.badw.de
}

\begin{abstract}
Superconducting quantum circuits are promising systems for experiments testing fundamental quantum mechanics on a macroscopic scale and for applications in quantum information processing. We report on the fabrication and characterization of superconducting flux qubits, readout dc SQUIDs, on-chip shunting capacitors, and high-quality coplanar waveguide resonators. Furthermore, we discuss the tunability and fundamental symmetry aspects inherent to all superconducting qubits, which can be regarded as artificial solid-state atoms. Comparing them to their natural counterparts, we discuss first and second-order energy shifts due to static control fields. Additionally, we present an intuitive derivation of the first- and second-order matrix elements for
\end{abstract} level transitions in the presence of a coherent microwave driving.

PACS numbers: 85.25.-j, 85.25.Cp 03.67.a

Submitted to: Supercond. Sci. Technol.

\section{Introduction}

Over the last decade the idea of solid-state based quantum information processing has excited scientists and engineers in many disciplines, seeking to combine two major developments of the last century, namely quantum mechanics and integrated circuit technology [1]. Although the practical challenges to realize solid-state based quantum information circuits are huge, the basic building blocks have been successfully implemented. Nevertheless, their fabrication and measurement remain demanding tasks. At present, most experiments are addressing two key questions. First, how can we fabricate, control, and read-out solid-state quantum circuits with sufficiently long coherence times, and second, how can we establish a controlled coupling between distant circuit parts and transfer the quantum information between them. Regarding the first problem, we focus on the fabrication of several key elements of superconducting quantum circuits. Their quantum coherent dynamics was discussed in our previous work $[2,3,4]$. With respect to the second one, we address symmetry aspects of these circuits. In particular, we discuss how symmetry can be broken in a controlled way using external control parameters [2]. 
In this context, superconducting circuits containing nonlinear elements such as Josephson junctions or phase-slip centers play an important role. The lateral dimensions of these circuits and their constituents can range from $100 \mathrm{~nm}$ to a few hundreds of $\mu \mathrm{m}$. Such circuits constitute artificial atoms in the sense that they behave similarly to natural atoms in many aspects [5]. Despite the fact that these artificial atoms are huge compared to their natural counterparts, they have a discrete level structure and exhibit properties unique to the world of quantum mechanics. In the simplest case, they form quantum two-level systems, also referred to as quantum bits or qubits. Such qubits are particularly attractive for the investigation of fundamental quantum phenomena on a macroscopic scale as well as for the use in quantum information systems. A major advantage of artificial solid-state atoms over natural atoms is their design flexibility and wide tunability by means of external parameters such as electric or magnetic fields. Coupling superconducting qubits to on-chip microwave resonators has given rise to circuit quantum electrodynamics (QED) $[6,7,8,9,10,11,12,13,14,15,16,17,18]$. In this prospering field, the fundamental interaction between artificial solid-state atoms (qubits) and (single) microwave photons is studied. We note that circuit QED is the solid-state analog of cavity QED $[19,20,21]$ in quantum optics, where natural atoms are coupled to suitable three-dimensional cavities. However, in contrast to quantum-optical cavity QED, the strong coupling regime, where the qubit-photon interaction coefficient is much larger than all relevant decay rates of qubit and resonator, can be achieved easily in circuit QED [7, 8]. Moreover, solid-state circuits exhibit an inherent tunability. We have recently shown that this can be exploited to break the symmetry of a superconducting flux qubit coupled to a lumped-element microwave resonator in a controlled way. This allowed us to study multi-photon excitations and to get fundamental insight into the interplay of multi-photon processes and symmetries in a qubit-resonator system [2]. In another work, we introduced a systematic formalism for two-resonator circuit QED, where two on-chip microwave resonators are simultaneously coupled to one superconducting qubit [18]. Within this framework, we demonstrated that the qubit can function as a quantum switch mediating a tunable interaction between the two resonators, which are assumed to be originally independent [18].

This article is composed as follows. In section 2, we focus on the fabrication of superconducting flux qubits, their readout circuitry and high-quality superconducting on-chip resonators. Then, in section 3, we address fundamental symmetry aspects of artificial solid-state atoms and compare them to their natural counterparts. As it turns out, these results hold for a general quantum two-level system [22]. In particular, we spell out the expressions for both flux and charge qubits.

\section{Fabrication of Superconducting Quantum Circuits}

Superconducting quantum circuits are based on flux quantization and Josephson tunneling. They have been studied intensively because they have opened a new area in fundamental science and because of their potential for the realization of quantum information processing systems [5]. Here, we discuss the fabrication process of superconducting three-junction flux qubits and the additional circuit elements required for readout, coupling and manipulation. 


\subsection{Flux Qubits}

Today, there are three fundamental types of superconducting qubits, namely flux, charge and phase qubits [5], depending on the nature of the relevant quantum variable. The three-junction flux qubit $[23,24,25]$, we are focussing on here, consists of a superconducting loop interrupted by three nm-sized Josephson junctions. Whereas two of these junctions with area $A$ and critical current $I_{\mathrm{c}}$ are equal, one junction is designed to have smaller area $\alpha A$ and critical current $\alpha I_{\mathrm{c}}$ with $\alpha \sim 0.6-0.8$. In an equivalent circuit the Josephson junctions are represented by a normal resistance $R_{\mathrm{n}}$, a junction capacitance $C_{\mathrm{J}}$ and a Josephson inductance $L_{\mathrm{J}}=L_{\mathrm{c}} / \cos \varphi$ with $L_{\mathrm{c}}=\hbar / 2 e I_{\mathrm{c}}$. Here, $\varphi$ is the phase difference across the junction, $\hbar$ the reduced Planck's constant, and $e$ the elementary charge. The characteristic energies are the Josephson coupling energy of the junctions, $E_{\mathrm{J}}=\hbar I_{\mathrm{c}} / 2 e$, associated with the storage of a single flux quantum $\Phi_{0}=h / 2 e$ in the Josephson inductor, and the charging energy, $E_{\mathrm{c}}=e^{2} / 2 C_{\mathrm{J}}$, associated with the storage of a single electron charge $e$ on the junction capacitance $C_{\mathrm{J}}$. For flux qubits, $E_{\mathrm{J}} \gg E_{\mathrm{c}}$ (typically $10 \leq E_{\mathrm{J}} / E_{\mathrm{c}} \leq 100$ ) to ensure that the magnetic flux $\Phi$ in the loop is the relevant quantum variable and the ground and the first excited state of the qubit have opposite circulating currents which produce measurable flux. The minimum temperature for experiments on superconducting qubits usually is limited to the base temperature $T_{\mathrm{b}} \gtrsim 10 \mathrm{mK}$ of dilution refrigerators. To avoid thermal population of the upper qubit state, the level splitting $\Delta_{\text {ge }}$ between the ground state $|\mathrm{g}\rangle$ and the first excited state $|\mathrm{e}\rangle$ should be much larger than $k_{\mathrm{B}} T_{\mathrm{b}}$, corresponding to an energy and frequency of about $1 \mu \mathrm{eV}$ and $200 \mathrm{MHz}$, respectively, at $T_{\mathrm{b}}=10 \mathrm{mK}$. Hence, $\Delta_{\text {ge }} / h \gtrsim 2 \mathrm{GHz}$ is desired. Since $\Delta_{\text {ge }} \propto \hbar \omega_{\mathrm{p}} \exp \left(-a \sqrt{E_{\mathrm{J}} / E_{\mathrm{c}}}\right)$, where $\omega_{\mathrm{p}} \propto E_{\mathrm{c}} \sqrt{E_{\mathrm{J}} / E_{\mathrm{c}}}$ is the plasma frequency and $a$ a constant of the order of unity, large $E_{\mathrm{c}}$ is required [23, 24]. At the same time one has to keep $E_{\mathrm{J}} / E_{\mathrm{c}}$ large enough to have well-defined flux states with a measurable circulating current. These requirements are demanding regarding junction fabrication technology, asking for junctions with high critical current densities $J_{\mathrm{c}} \simeq 10^{3} \mathrm{~A} / \mathrm{cm}^{2}$ and areas down to a few $0.01 \mu \mathrm{m}^{2}$. Since such small junctions with small parameter spread are difficult to fabricate by the well established $\mathrm{Nb} / \mathrm{AlO}_{x} / \mathrm{Nb}$ technology, most flux qubits so far are based on $\mathrm{Al} / \mathrm{AlO}_{x} / \mathrm{Al}$ junctions fabricated by electron beam lithography and two-angle shadow evaporation [26].

Figure 1(a) shows a scanning electron microscopy (SEM) micrograph of a threejunction flux qubit based on $\mathrm{Al} / \mathrm{AlO}_{x} / \mathrm{Al}$ junctions. The qubit is surrounded by the readout dc SQUID (Superconducting Quantum Interference Device). Figures 1(d) and (e) show an enlarged view of the so-called $\alpha$-junction, which has a reduced area $\alpha A$ with $\alpha=0.7$, and one of the regular qubit junctions, respectively. The area of the $\alpha$-junction is only about $0.02 \mu \mathrm{m}^{2}$. The qubits are fabricated on thermally oxidized $\left(50 \mathrm{~nm} \mathrm{SiO}_{2}\right)$ Si wafers by electron beam lithography using a Philips XL30 SFEG field emission SEM and a Raith Elphy Plus nanolithography system. For producing freestanding resist masks we used a double-layer resist system. Typical cross-sectional views of the resist stencils are shown in Figs. 1(b) and (c). Due to the larger sensitivity of the underlay resist, large undercuts can be generated in a controlled way. The two-angle shadow evaporation of $\mathrm{Al}$ was done by electron beam evaporation in a UHV system with a base pressure in the $10^{-9} \mathrm{mbar}$ range. The bottom layer was thermally oxidized in situ in pure oxygen at $p=2 \times 10^{-4}$ mbar. In order to achieve high current densities we used small products $L$ of oxygen pressure and oxidation time ranging between 0.25 and $0.26 \mathrm{mbar} \cdot \mathrm{s}$. With these values we achieved 

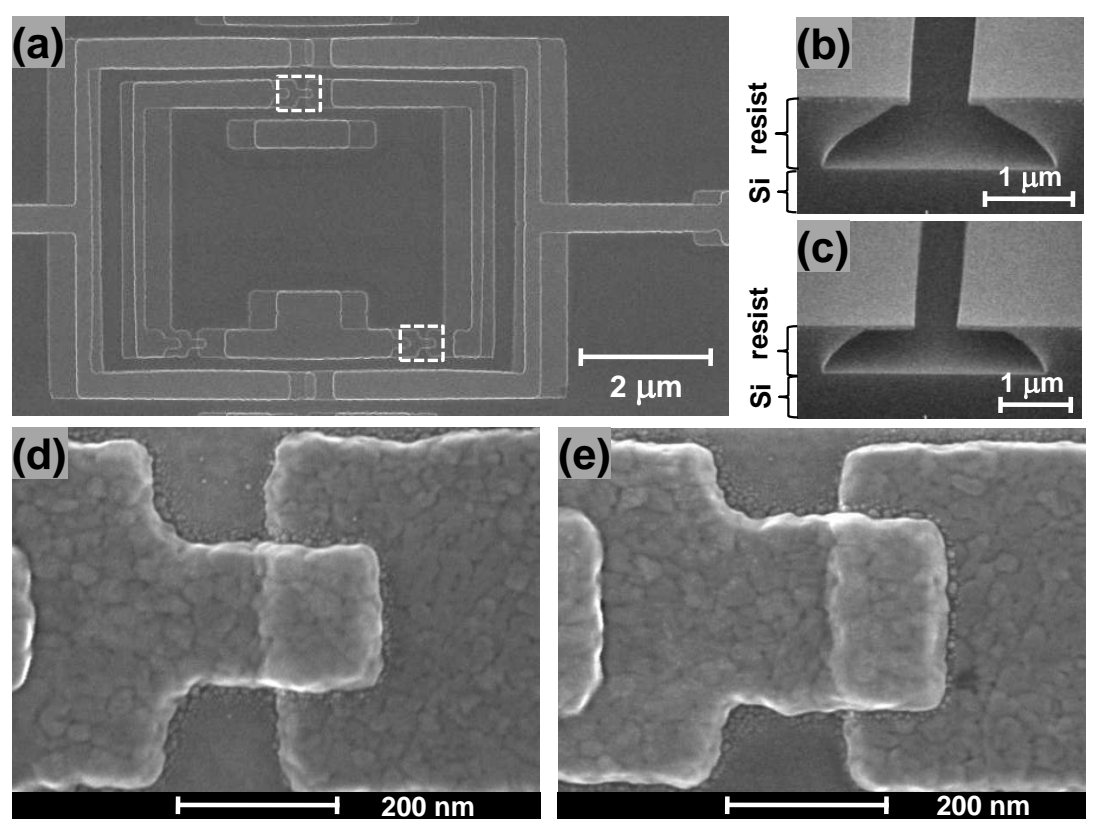

Figure 1. (a) SEM micrograph of a three-junction flux qubit (inner loop) surrounded by the readout dc SQUID (outer loop with two junctions). The white rectangles mark the regions of the $\alpha$-junction (upper) with a reduced area $(\alpha=0.7)$ and a regular junction (lower) shown on an enlarged scale in (d) and (e), respectively. (b) and (c) show cross-sectional views of doublelayer resist structures obtained with different exposure times. Large undercuts can be obtained, resulting in free-standing resist parts required for the shadow evaporation technique.

$J_{\mathrm{c}}(50 \mathrm{mK}) \simeq 1500 \pm 500 \mathrm{~A} / \mathrm{cm}^{2}$ with a high yield of $85 \%$. By optimizing the electron beam lithography process we could reduce the spread in the junction area down to $10 \%$. We note that we can increase or decrease $J_{\mathrm{c}}$ by decreasing or increasing $L$, respectively. For example, by increasing $L$ to above $10 \mathrm{mbar} \cdot \mathrm{s}$ the $J_{\mathrm{c}}$ values can be reduced to below $10 \mathrm{~A} / \mathrm{cm}^{2}$.

Figure 2 shows the current-voltage characteristics (IVC) and the flux dependence of the critical current of a readout dc SQUID fabricated with the process described above. From $I_{\mathrm{c}}=1.44 \mu \mathrm{A}$ and the measured junction area $A=0.035 \mu \mathrm{m}^{2}$ we obtain $J_{\mathrm{c}}=2 \mathrm{kA} / \mathrm{cm}^{2}$. Furthermore, from $R_{\mathrm{n}}=169 \Omega$ the product $I_{\mathrm{c}} R_{\mathrm{n}}=243 \mu \mathrm{V}$ is obtained. The measured gap voltage $V_{\mathrm{g}} \simeq 360 \mu \mathrm{V}$ is close to the BCS value $V_{\mathrm{g}}=2 \Delta / e=3.53 k_{\mathrm{B}} T_{\mathrm{c}} / e=365 \mu \mathrm{V}$ using $T_{\mathrm{c}}=1.2 \mathrm{~K}$ for the critical temperature of Al. Furthermore, the measured $I_{\mathrm{c}} R_{\mathrm{n}}$ product agrees well with the AmbegaokarBaratoff value [27] $\pi V_{\mathrm{g}} / 4=287 \mu \mathrm{V}$. Using the specific junction capacitance $C_{\mathrm{s}}=$ $100 \pm 25 \mathrm{fF} / \mu \mathrm{m}^{2}$ [28], the capacitance of the qubit junctions with area $A=0.02 \mu \mathrm{m}^{2}$ is estimated to be $C_{\mathrm{J}} \simeq 2 \mathrm{fF}$ corresponding to $E_{\mathrm{c}}=40 \mu \mathrm{eV}$ or, equivalently, $T=0.46 \mathrm{~K}$. The subgap structures in the IVC in Figure 2(a) most likely originate from $L C$ resonances as has been discussed in more detail recently [28].

We note that the fabrication of sub-micron sized Josephson junctions with twoangle shadow evaporation is very common since it is easy to use. However, there are also drawbacks. In particular, the reproducibility and parameter spread of the 

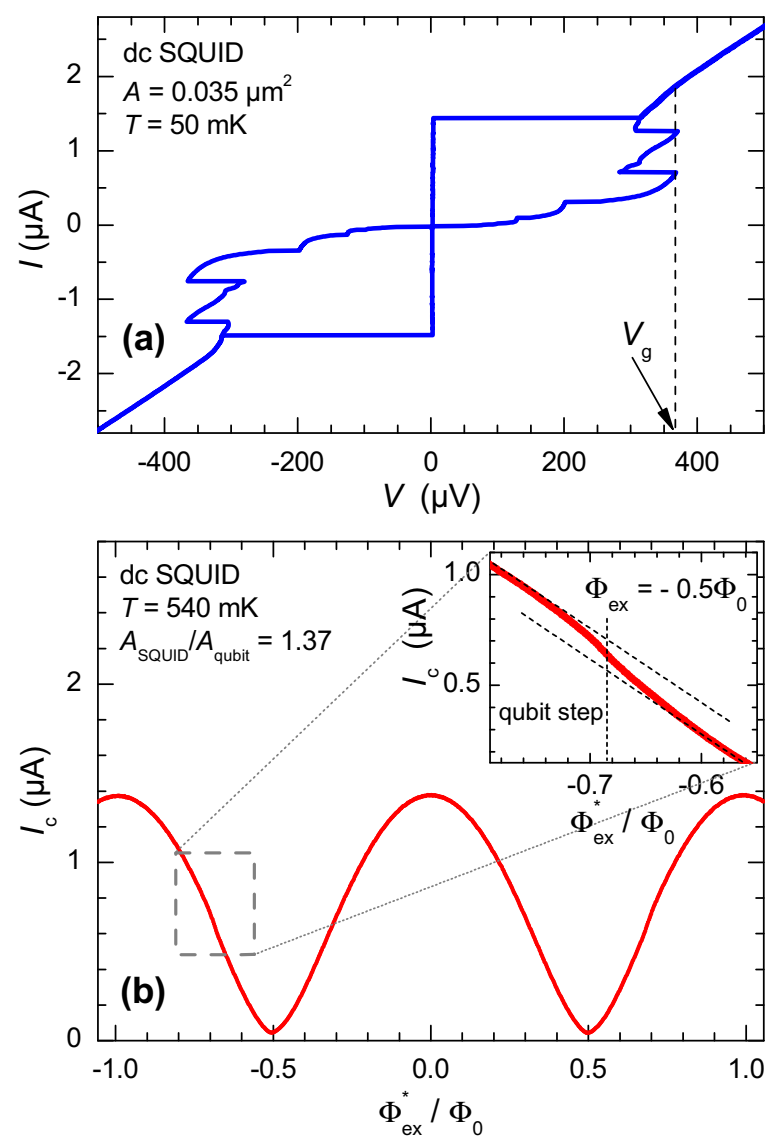

Figure 2. (a) Current-voltage characteristics of a readout dc SQUID based on $\mathrm{Al} / \mathrm{AlO}_{x} / \mathrm{Al}$ tunnel junctions with $A=0.035 \mu \mathrm{m}^{2}$ at $T=50 \mathrm{mK}$. (b) Critical current $I_{\mathrm{c}}$ of a readout dc SQUID as a function of the applied magnetic flux $\Phi_{\mathrm{ex}}^{\star}$ threading the SQUID loop at $T=540 \mathrm{mK}$. The inset shows an enlarged view of the region around $\Phi_{\mathrm{ex}}=-\gamma \Phi_{0} / 2$, where the qubit persistent current changes sign. Here, $\gamma=1.37$ is the ratio of the loop areas of the SQUID and the qubit.

junctions will be finally limited by the non-planar junction geometry, resulting in a not well defined junction area. The related fluctuations in the critical current become an increasing problem when one is going to more complex quantum circuits. However, more severe is critical current noise due to impurities and trapping sites in the tunneling barrier leading to decoherence. Therefore, in the long run a planar junction geometry and the use of epitaxial electrode and barrier layers may be the optimum choice.

The $I_{\mathrm{c}}\left(\Phi_{\mathrm{ex}}^{\star}\right)$ dependence of a readout dc SQUID is shown in Fig. 2(b). The measured curve is close to the ideal $\left|\cos \left(\pi \Phi_{\text {ex }}^{\star} / \Phi_{0}\right)\right|$ dependence expected for a dc SQUID with $\beta_{\mathrm{L}}=2 \pi L_{\mathrm{s}} I_{\mathrm{c}} / \Phi_{0}=L_{\mathrm{s}} / L_{\mathrm{c}} \ll 1$, for which the Josephson inductance $L_{\mathrm{c}}$ dominates the geometric inductance $L_{\mathrm{s}}$. However, there are small deviations, originating from the additional flux due to the persistent current $I_{\mathrm{p}}$ circulating clockor counterclockwise in the qubit loop. In the region close to the applied flux $\Phi_{\text {ex }}$ corresponding to $\Phi_{0} / 2$ in the qubit loop, $I_{\mathrm{p}}$ changes sign. This results in a step-like 

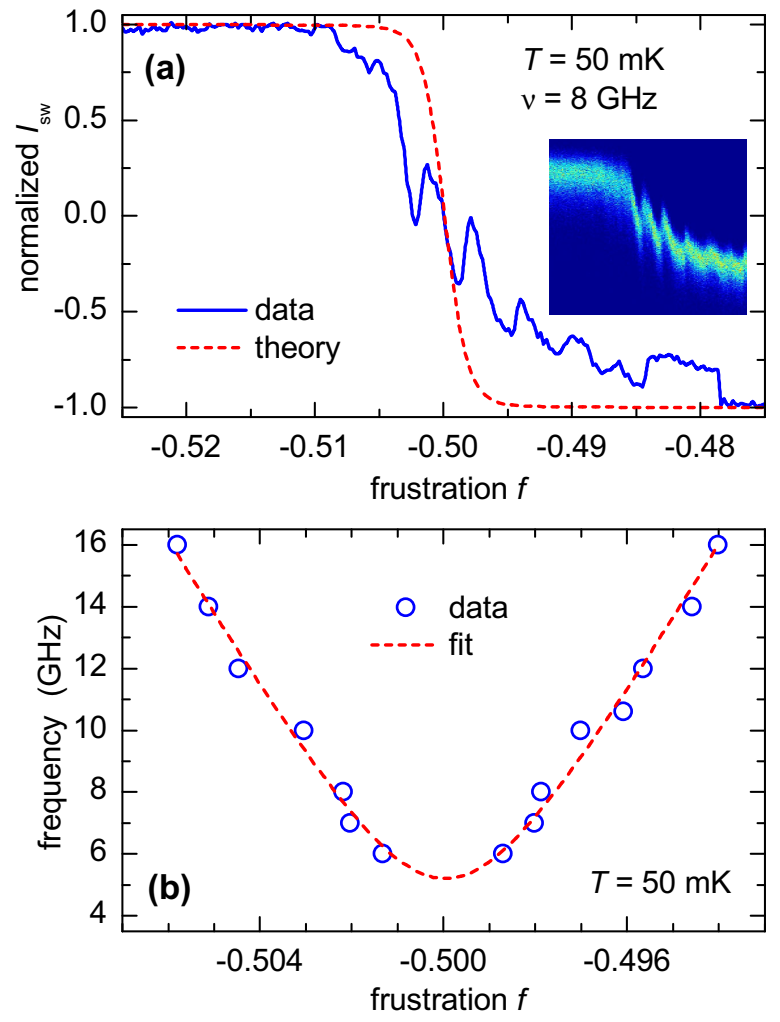

Figure 3. (a) Normalized switching current $I_{\mathrm{sw}}$ of the readout dc SQUID plotted versus the frustration $f=\Phi_{\mathrm{ex}} / \Phi_{0}$ in the region around the qubit step at $T=$ $50 \mathrm{mK}$. The qubit is irradiated by a microwave signal $(\nu=8 \mathrm{GHz})$. The dashed curve shows the theoretically expected curve without microwave irradiation for $\Delta_{\text {ge }} / h=5.2 \mathrm{GHz}$. The inset shows the switching current distribution in a color coded image. (b) Qubit transition frequency $E_{\text {ge }} / h$ (symbols) plotted versus the frustration $f$. The line is a numerical fit to the data yielding $\Delta_{\text {ge }} / h=5.2 \mathrm{GHz}$ and $I_{\mathrm{p}}=450 \mathrm{nA}$.

feature superimposed on the regular $I_{\mathrm{c}}\left(\Phi_{\mathrm{ex}}^{\star}\right)$ dependence. This so-called qubit step is shown in the inset of Fig. 2(b). With the ratio of 1.37 between the SQUID and the qubit loop we estimate $\Phi_{\mathrm{ex}}=-0.685 \Phi_{0}$ in good agreement with the experiment.

The qubit level structure has been investigated by microwave spectroscopy. Figure 3(a) shows the normalized switching current of the readout SQUID around the qubit step plotted versus the frustration $f=\Phi_{\text {ex }} / \Phi_{0}$. Here, $\Phi_{\text {ex }}$ is the applied flux threading the qubit loop. Note that the switching of the SQUID from the zerovoltage into the voltage state is by quantum tunneling and hence is a statistical process. Therefore, the normalized switching current $I_{\mathrm{sw}}(f)$ is obtained from the switching current distribution measured for every $f$. This distribution is shown in a color-coded image in the inset of Fig. 3(a). The $I_{\mathrm{sw}}(f)$ curve shows a pronounced peak-dip structure due to resonant transitions between the qubit levels induced by the applied microwave irradiation with frequency $\nu=8 \mathrm{GHz}$. Note that multi-photon transitions are observed depending on the applied microwave power. The position of the peaks/dips (one-photon transitions) as a function of frequency and frustration is 

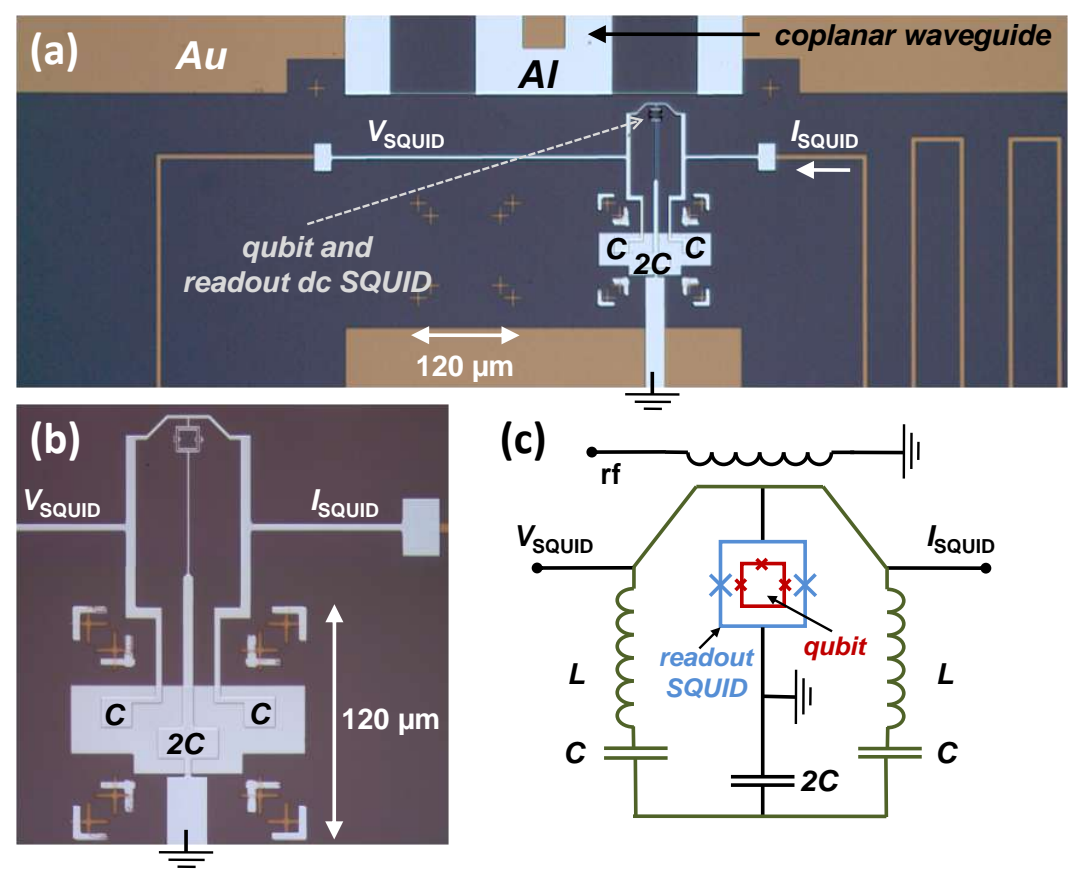

Figure 4. (a) Optical micrograph of the readout and bias circuit of the dc SQUID and the end of the coplanar waveguide which is shorted to ground to form an antenna. In (b) an enlarged view of the region around the qubit and SQUID loop with the shunting capacitors is shown. The equivalent circuit is depicted in (c).

shown in Fig. 3(b). The data follow the expected $E_{\text {ge }}=\sqrt{\epsilon^{2}+\Delta_{\text {ge }}^{2}}$ dependence, where $\epsilon=2 I_{\mathrm{p}} \Phi_{0}(f+0.5)$ is the energy bias and $\Delta_{\text {ge }}$ the level splitting at the degeneracy point $f=-0.5$. Fitting the data yields $I_{\mathrm{p}}=450 \mathrm{nA}$ and a qubit gap of $\Delta_{\text {ge }} / h=5.2 \mathrm{GHz}$.

\subsection{Bias and Readout Circuit}

For the manipulation and readout of flux qubits as well as for the attenuation of external electrical and magnetic noise additional circuit elements such as resistors, capacitors, inductors, microwave lines, antennas etc. are required. Some of them have to be fabricated on-chip. For the fabrication of these usually larger circuit parts we used a mix\&match process, where high resolution electron beam lithography patterns are precisely aligned into existing patterns made by optical lithography. For the alignment markers, the leads and the coplanar microwave lines we used $20 \mathrm{~nm}$ thick $\mathrm{Au}$ layers. These layers are fabricated by optical lithography, electron beam evaporation and a final lift-off process prior to the e-beam process for the qubits described above. During the subsequent $\mathrm{Al}$ evaporation process, parts of the Au pattern are covered by Al. The small thickness of the Au layer allows to avoid the interruption of the $\mathrm{Al}$ film at the edges of the Au layer.

Figures 4(a) and (b) show optical micrographs of the circuit elements used for biasing the readout dc SQUID and measuring the SQUID voltage. As shown by the equivalent circuit in Fig. 4(c), the SQUID is shunted to ground by on-chip $\mathrm{Al} / \mathrm{AlO}$ / $/ \mathrm{Al}$ capacitors with the total capacitance $C$. In combination with the on-chip resistors 
in the dc SQUID bias and voltage lines, which are realized by long Au lines, they form a low-pass filter constituting the main component of the qubit electromagnetic environment. Details on the shaping of the electromagnetic environment as well as the effect of a resistive and capacitive coupling of the dc SQUID are given elsewhere $[4,3]$. The ground plane of the $\mathrm{Al} / \mathrm{AlO}_{x} / \mathrm{Al}$ capacitors is deposited by electron beam evaporation prior to the qubit fabrication process. It is thermally oxidized in ambient atmosphere for 3 hours at $100^{\circ} \mathrm{C}$ to achieve a thick oxide layer with sufficiently small leakage current. Specific capacitance values between 12 and $15 \mathrm{fF} / \mu \mathrm{m}^{2}$ and resistance times area products larger than $100 \mathrm{G} \Omega \mu \mathrm{m}^{2}$ could be achieved.

It is evident from Fig. 4(c) that the two capacitors with total capacitance $C / 2$ together with the two inductors with total inductance $2 L$ form an $L C$ resonator coupled inductively to the qubit loop. The typical resonance frequency $\nu_{\mathrm{r}}=\omega_{\mathrm{r}} / 2 \pi$ of the $L C$ resonator is in the $\mathrm{GHz}$ range and is comparable to the qubit transition frequency. Furthermore, the coupling strength $g / 2 \pi$ is of the order of $100 \mathrm{MHz}[2,11]$. Therefore, the coupled qubit-resonator system allows to study fundamental questions of the flourishing field of circuit QED [7, 8, 11, 12, 13, 14, 15, 29]. Here, the interaction of solid-state quantum systems with single microwave photons is of particular interest. Recently, we have used a circuit very similar to that shown in Fig. 4 to observe one of the key signatures of a two-photon driven Jaynes-Cummings model, which unveils the upconversion dynamics of a superconducting flux qubit coupled to an on-chip lumped-element microwave resonator [2].

\subsection{Microwave Resonators}

The quality factor $Q_{\mathrm{L}}$ of the $L C$ resonators discussed above is only of the order of 100. Therefore, the decay rate $\kappa=\nu_{\mathrm{r}} / Q_{\mathrm{L}}$ of the microwave photons from the resonator is large. In order to access the strong coupling regime in circuit QED experiments, $g / 2 \pi \gg \kappa$ is required. With $g / 2 \pi \sim 100 \mathrm{MHz}$ and $\omega_{\mathrm{r}} / 2 \pi \sim 10 \mathrm{GHz}$, microwave resonators with $Q_{\mathrm{L}} \gtrsim 10^{4}$ have to be designed. Such resonators can be realized by simple transmission line geometries [30].

We have fabricated coplanar waveguide (CPW) resonators based on $200 \mathrm{~nm}$ thick $\mathrm{Nb}$ films on thermally oxidized $\mathrm{Si}$ and sapphire substrates. An optical micrograph of such a resonator is shown in Fig. 5(a). The Nb films have been deposited by dc magnetron sputtering in Ar. The patterning was done by optical lithography and reactive ion etching using an $\mathrm{Ar} / \mathrm{SF}_{6}$ mixture. The center conductor of the $\mathrm{CPW}$ resonators is $20 \mu \mathrm{m}$ wide, separated from the lateral ground planes by a $12 \mu \mathrm{m}$ wide gap to obtain a wave impedance of $50 \Omega$. The resonator is coupled at both ends to a coplanar transmission line by interdigital or gap capacitors shown in the insets of Fig. 5(a). The strength of the capacitive coupling determines the loaded quality factor $Q_{\mathrm{L}}^{-1}=Q^{-1}+Q_{\text {ext }}^{-1}$, where $Q$ is the internal quality factor of the uncoupled resonator and $Q_{\text {ext }}$ is determined by the value of the coupling capacitors $C_{\text {ext }}$ and the load impedance ( $50 \Omega$ in our case). By proper choice of $C_{\text {ext }}$ the loaded quality factor can be designed to an optimum value satisfying on the one hand the condition $g \gg \kappa$ and allowing on the other hand an as high as possible measuring rate $(\propto \kappa)$. The loaded quality factor $Q_{\mathrm{L}}$ has been extracted from $S_{21}$ measurements as shown in Fig. 5(d). In Fig. 5(c) we have plotted $Q_{\mathrm{L}}$ as a function of the coupling capacitance $C_{\text {ext }}$, calculated

using FastCap ${ }^{\circledR}$. The measured dependence nicely follows the theoretical prediction [31] showing that $Q_{\mathrm{L}}$ can be deliberately designed over a wide range. As shown in Fig. 5(d), internal quality factors $Q>10^{5}$ can be achieved with the niobium CPW 

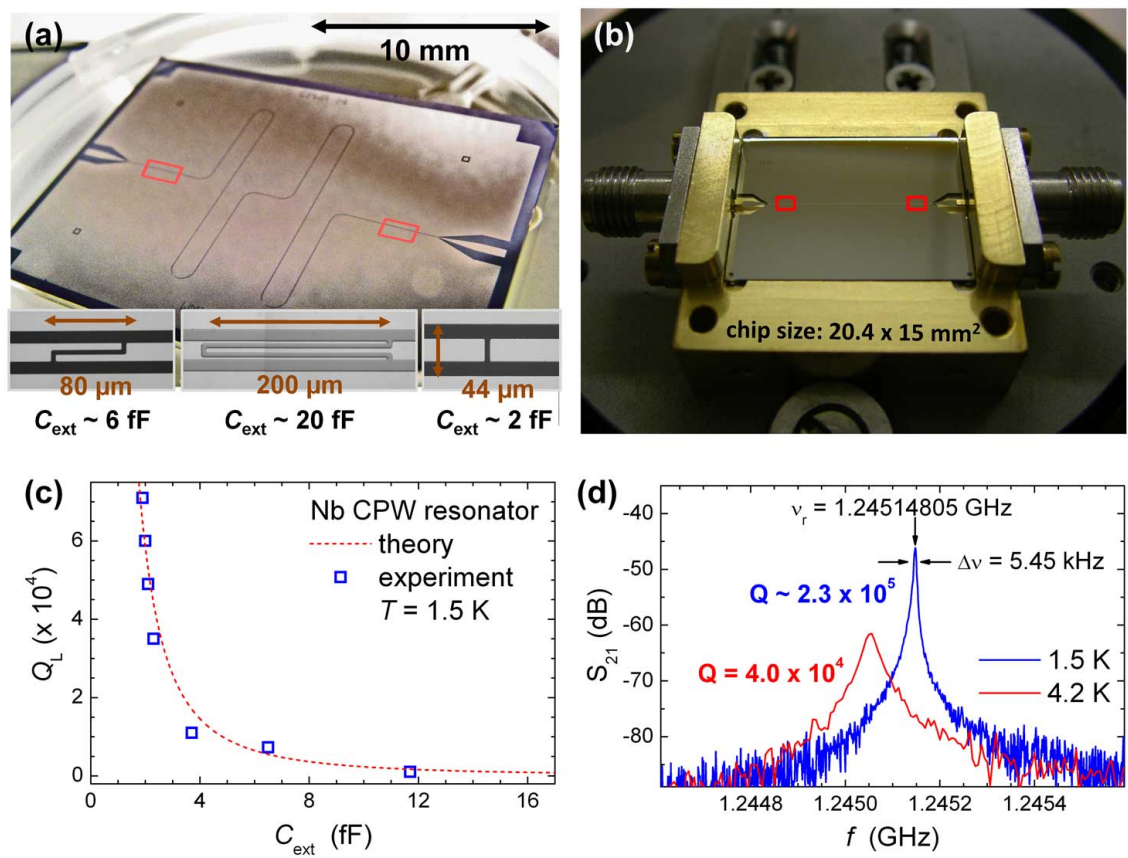

Figure 5. (a) optical micrograph of a CPW microwave resonator. The insets show different coupling capacitors located at the positions marked by the rectangles. (b) Picture of the gold-plated copper sample box containing a resonator chip. The rectangles mark the positions of the coupling capacitors. (c) Measured $Q_{\mathrm{L}}$ (symbols) plotted versus the coupling capacitance for a $6 \mathrm{GHz}$ niobium CPW resonator at $T=1.5 \mathrm{~K}$. The dashed line shows the theoretical prediction [31]. (d) Transmission spectrum of an undercoupled 1.25 GHz niobium $\mathrm{CPW}$ resonator measured at 1.5 and $4.2 \mathrm{~K}$.

resonators already at $1.5 \mathrm{~K}$.

\section{Tunability, Coupling Strength and Symmetry Breaking in Superconducting Circuit QED}

The field of superconducting circuit QED has attracted considerable attention over the last years, since it allows to study the interaction of microwave photons and solidstate quantum circuits on a quantum level. In the following we consider a classically driven quantum system [32] consisting of a qubit coupled to a harmonic oscillator. We note that the subsequent discussion is quite general. It is not restricted to the flux qubits discussed in section 2, but is valid for a general quantum two-level system including both superconducting flux and charge qubits. The first-order hamiltonian of this system in the diabatic basis of the qubit eigenstates $\{|\downarrow\rangle,|\uparrow\rangle\}$ is given by $[2,9]$

$$
\begin{aligned}
\widehat{H}= & \frac{\epsilon}{2} \widehat{\sigma}_{z}-\frac{\Delta_{\mathrm{ge}}}{2} \widehat{\sigma}_{x}+\hbar \omega_{\mathrm{r}}\left(\hat{a}^{\dagger} \hat{a}+\frac{1}{2}\right) \\
& +\hbar g \widehat{\sigma}_{z}\left(\hat{a}^{\dagger}+\hat{a}\right)+\frac{\Omega}{2} \widehat{\sigma}_{z} \cos \omega t+\eta\left(\hat{a}^{\dagger}+\hat{a}\right) \cos \omega t .
\end{aligned}
$$


Here, the Hamiltonian $\widehat{H}_{\mathrm{q}} \equiv(\epsilon / 2) \widehat{\sigma}_{z}-\left(\Delta_{\text {ge }} / 2\right) \widehat{\sigma}_{x}$ describes the qubit and $\widehat{H}_{\mathrm{r}} \equiv$ $\hbar \omega_{\mathrm{r}}\left(\hat{a}^{\dagger} \hat{a}+1 / 2\right)$ represents a quantum harmonic oscillator with the photon number states $|0\rangle,|1\rangle,|2\rangle, \ldots \hat{a}^{\dagger}$ and $\hat{a}$ are the boson creation and annihilation operators and $\widehat{\sigma}_{x}$ and $\widehat{\sigma}_{z}$ the Pauli operators. The level splitting at the degeneracy point $\Delta_{\text {ge }}>0$ by definition. The interaction term between qubit and resonator is $\widehat{H}_{\mathrm{q}, \mathrm{r}}=\hbar g \widehat{\sigma}_{z}\left(\hat{a}^{\dagger}+\hat{a}\right)$, and $\widehat{H}_{\mathrm{m}, \mathrm{q}}=(\Omega / 2) \widehat{\sigma}_{z} \cos \omega t$ and $\widehat{H}_{\mathrm{m}, \mathrm{r}}=\eta\left(\hat{a}^{\dagger}+\hat{a}\right) \cos \omega t$ describe the qubit and the resonator microwave driving, respectively. Furthermore, $\omega_{\mathrm{r}} / 2 \pi$ is the fundamental resonator frequency, $g / 2 \pi$ the qubit-resonator coupling strength, and $\Omega$ and $\eta$ are the qubit and the resonator driving strength. At the qubit optimal point and, under some approximations, also away from it, the hamiltonian (1) is equivalent to that describing a driven system consisting of an atom coupled to the light field in an optical cavity. Therefore, solid-state circuit QED can be considered the circuit analogue of cavity QED. We note that the hamiltonian (1) can be applied to both flux and charge qubits by choosing an $\epsilon=2 I_{\mathrm{p}} \delta \Phi_{\mathrm{ex}}$ and $\epsilon=2 V_{\mathrm{p}} \delta Q_{\mathrm{ex}}$, respectively. Here, $V_{\mathrm{p}}=e / C_{\mathrm{g}}$ is the voltage associated with an elementary charge on the gate capacitance $C_{\mathrm{g}}, \delta \Phi_{\mathrm{ex}}$ and $\delta Q_{\text {ex }}$ are the applied gate flux and charge relative to the degeneracy point. The latter is located at half a flux quantum and half a Cooper pair charge, respectively. In general, one can switch between the expressions for flux and charge qubits by replacing the quantities current, flux and inductance by voltage, charge and capacitance.

Most interesting in circuit QED is the so-called strong coupling regime, $g / 2 \pi \gg$ $\kappa, \gamma$, where $\kappa$ is the resonator decay rate and $\gamma$ is the largest of the qubit decay rates. In this situation, the reversible exchange of microwave photons between a solid-state twolevel quantum system (qubit) and a resonator represents a coherent oscillatory process. Regarding quantum information processing, this means that quantum information can be exchanged back and forth many times. To achieve the strong coupling regime, the qubit-resonator coupling strength $g / 2 \pi$ must be of the order of $100 \mathrm{MHz}$ because presently $\gamma \gtrsim 1 \mathrm{MHz}$, i.e., the decay rate of the qubit into other channels is still large for superconducting qubits. As demonstrated in section 2, the fabrication of suitable resonators with $\kappa<1 \mathrm{MHz}$ is possible. Compared to optical cavity QED, such large values for $g / 2 \pi$ can be obtained more easily in superconducting circuit QED for two reasons. First, the electric and magnetic dipole moments, $\mu_{\mathrm{el}}$ and $\mu_{\text {mag }}$, of superconducting charge and flux qubits, respectively, are much larger than those of natural atoms. Second, the mode volume $V_{\mathrm{m}}$ of transmission line microwave resonators (cf. Fig. 5) can be made much smaller than for 3D cavities in quantum optics, resulting in large electric or magnetic fields. Note that the fields associated with vacuum fluctuations are $E_{0}=\sqrt{\hbar \omega_{\mathrm{r}} / 2 \epsilon_{0} V_{\mathrm{m}}}$ and $B_{0}=\sqrt{\mu_{0} \hbar \omega_{\mathrm{r}} / 2 V_{\mathrm{m}}}$. Since mode volumes as small as $10^{-12} \mathrm{~m}^{3}$ seem to be feasible, the electric and magnetic fields associated with vacuum fluctuations are of the order of $0.1 \mathrm{~V} / \mathrm{m}$ and $1 \mathrm{nT}$ for $\omega_{\mathrm{r}} / 2 \pi$ of a few $\mathrm{GHz}$.

In the following we first address the tunability of the energy levels of superconducting charge and flux qubits by static electric and magnetic fields. The derived energy shifts are equivalent to the dc Stark and Zeeman shifts in natural atoms. Then, we discuss the atom-resonator coupling strength and some fundamental symmetry aspects of superconducting circuit QED. In our discussion we use a semiclassical approach, treating the microwave driving as classical fields. 


\subsection{Tunability of Charge and Flux Qubits}

In this section we compare artificial superconducting atoms (charge and flux qubits) to natural atoms (we use hydrogen for simplicity) regarding their tunability by static fields. In Table 1 we have summarized the first- and second-order energy shifts $\Delta E_{\mathrm{el}, \mathrm{mag}}^{(1)}$ and $\Delta E_{\mathrm{el}, \mathrm{mag}}^{(2)}$ due to electric and magnetic fields, $\delta E_{\mathrm{ex}}$ and $\delta B_{\mathrm{ex}}$, applied via suitable control gates. Discussing the first-order terms, we consider the charge qubit at the degeneracy point as a system of two degenerate levels $|\downarrow\rangle$ and $|\uparrow\rangle$ corresponding to adjacent Cooper-pair number states with Cooper pair numbers $N$ and $N+1$. At this point, the polarization charge $Q_{\mathrm{ex}}=2 e N_{\mathrm{ex}}$ induced by an applied gate voltage corresponds to $2 e(N+1 / 2)$ for both charge basis states. We define $\delta N_{\mathrm{ex}}=N_{\mathrm{ex}}-(N+1 / 2)$ as the deviation from the Cooper pair number at the degeneracy point. Therefore, at the degeneracy point we have $\delta N_{\mathrm{ex}}=0$ and, correspondingly, $\delta E_{\mathrm{ex}}=0$, where $\delta E_{\mathrm{ex}}=\delta Q_{\mathrm{ex}} / C_{\mathrm{g}} d=2 e \delta N_{\mathrm{ex}} / C_{\mathrm{g}} d$ is the electric field applied via a control gate, which just shifts the Cooper pair number on the island by $\delta N_{\text {ex }}$ away from the degeneracy point. Here, $C_{\mathrm{g}}$ is the gate capacitor and $d$ the distance between the control gate and the island. Our discussion shows that we can consider the charge qubit at the degeneracy point as a degenerate two-level system in a zero effective control electric field $\delta E_{\mathrm{ex}}=0$. The finite coupling of the charge states $|\downarrow\rangle$ and $|\uparrow\rangle$ results in the symmetric and antisymmetric superposition states $|\mathrm{g}\rangle=(|\downarrow\rangle+|\uparrow\rangle) / \sqrt{2}$ and $|\mathrm{e}\rangle=(|\downarrow\rangle-|\uparrow\rangle) / \sqrt{2}$, respectively, with an energy splitting $\Delta_{\text {ge }}$ of their eigenenergies. The situation for the flux qubit is completely analogous. At the degeneracy point it can be considered as a system of two degenerate levels $|\downarrow\rangle$ and $|\uparrow\rangle$ corresponding to the persistent currents $\pm I_{\mathrm{p}}$ circulating in the qubit loop. The polarization flux $\Phi_{\mathrm{ex}}=N_{\mathrm{ex}} \Phi_{0}$ is generated by a magnetic field $B_{\mathrm{ex}}$ applied via a control gate. Again, at the degeneracy point we have $\delta N_{\mathrm{ex}}=0$ and $\delta B_{\mathrm{ex}}=0$. Here, $\delta B_{\mathrm{ex}}=\delta \Phi_{\mathrm{ex}} / A=\delta N_{\mathrm{ex}} \Phi_{0} / A$ is the magnetic control field, which just changes the flux number in the loop of area $A$ by $\delta N_{\text {ex }}$. Evidently, at the degeneracy point we can consider the flux qubit as a two-level system in a zero effective magnetic control field $\delta B_{\text {ex }}=0$. As for the charge qubit, the finite coupling of the flux states results in superposition states with eigenenergies separated by $\Delta_{\text {ge }}$.

We now can discuss the interaction energy of the charge and flux qubit with static control fields $\delta F_{\text {ex }}=\left\{\delta E_{\mathrm{ex}}, \delta B_{\mathrm{ex}}\right\}$. Since both the electric and magnetic dipole moments in the two basis states $|\downarrow\rangle$ and $|\uparrow\rangle$ have opposite sign, the interaction operator in the basis $\{|\downarrow\rangle,|\uparrow\rangle\}$ can be written as

$$
\widehat{W}=-\mu \delta F_{\text {ex }} \widehat{\sigma}_{z}
$$

with the dipole moments $\mu=\left\{\mu_{\mathrm{el}}, \mu_{\mathrm{mag}}\right\}$ given by

$$
\begin{aligned}
& \mu_{\mathrm{el}}=e d \\
& \mu_{\mathrm{mag}}=I_{\mathrm{p}} A .
\end{aligned}
$$

We immediately see that the first-order energy shifts of the uncoupled levels $|\downarrow\rangle$ and $|\uparrow\rangle$ are given by the products

$$
\begin{aligned}
& \Delta E_{\mathrm{el}}^{(1)}= \pm \mu_{\mathrm{el}} \cdot \delta E_{\mathrm{ex}} \\
& \Delta E_{\mathrm{mag}}^{(1)}= \pm \mu_{\mathrm{mag}} \cdot \delta B_{\mathrm{ex}} .
\end{aligned}
$$

For natural atoms, the corresponding energy shifts are the linear Stark and Zeeman shifts. Note that natural atoms and molecules with an inversion center in a nondegenerate electronic state do not have a permanent electric dipole moment and 
Table 1. Characteristic first- and second-order shifts of the energy levels due to external electric and magnetic fields $\delta E_{\text {ex }}$ and $\delta B_{\text {ex }}$ for natural atoms (hydrogen) compared to those of superconducting charge and flux qubits. For the estimates of the electric and magnetic dipole moments $\mu_{\mathrm{el}}$ and $\mu_{\mathrm{mag}}$ as well as the polarizabilities $\chi_{\mathrm{el}}$ and $\chi_{\mathrm{mag}}$ we used the typical values $d=0.1-1 \mu \mathrm{m}$ for the distance between island and gate electrode (charge qubit), as well as $A=5-10 \mu \mathrm{m}^{2}$ and $I_{\mathrm{p}}=100-500 \mathrm{nA}$ for the loop area and the persistent current (flux qubit), respectively. Furthermore, $\mu_{0}=1.26 \times 10^{-6} \mathrm{Vs} / \mathrm{Am}$ is the vacuum permeability, $\epsilon_{0}=8.85 \times 10^{-12} \mathrm{As} / \mathrm{Vm}$ the vacuum permittivity, $a_{\mathrm{B}}=5.29 \times 10^{-11} \mathrm{~m}$ the Bohr radius, $m=9.109 \times 10^{-31} \mathrm{~kg}$ the electron mass, and $e=1.602 \times 10^{-19}$ As the electron charge.

\begin{tabular}{lccc}
\hline $\begin{array}{l}\text { energy } \\
\text { shift }\end{array}$ & natural atom & charge qubit & flux qubit \\
\hline$\Delta E_{\mathrm{el}}^{(1)}$ & $\pm \mu_{\mathrm{el}} \cdot \delta E_{\mathrm{ex}}$ & $\pm \mu_{\mathrm{el}} \cdot \delta E_{\mathrm{ex}}$ & - \\
& $\left\langle\mu_{\mathrm{el}}\right\rangle=0$ & $\mu_{\mathrm{el}}=e d$ & \\
\hline$\Delta E_{\mathrm{mag}}^{(1)}$ & $\pm \mu_{\mathrm{mag}} \cdot \delta B_{\mathrm{ex}}$ & $10^{-26}-10^{-25} \mathrm{Cm}$ & $\pm \mu_{\mathrm{mag}} \cdot \delta B_{\mathrm{ex}}$ \\
& $\mu_{\mathrm{mag}} \simeq \mu_{\mathrm{B}}$ & - & $\mu_{\mathrm{mag}}=I_{\mathrm{p}} A$ \\
& $-\frac{1}{2} \chi_{\mathrm{el}} \cdot \delta E_{\mathrm{ex}}^{2}$ & $-\frac{1}{2} \chi_{\mathrm{el}} \cdot \delta E_{\mathrm{ex}}^{2}$ & $\sim 10^{4}-10^{5} \mu_{\mathrm{B}}$ \\
\hline$\Delta E_{\mathrm{el}}^{(2)}$ & $\chi_{\mathrm{el}} \simeq 4 \pi \epsilon_{0} a_{\mathrm{B}}^{3}$ & $\chi_{\mathrm{el}} \simeq-2 \frac{(e d)^{2}}{\Delta \mathrm{ge}}$ & \\
& $\sim 10^{-41} \mathrm{Cm}^{2} / \mathrm{V}$ & $\sim 10^{-28}-10^{-26} \mathrm{Cm}^{2} / \mathrm{V}$ & \\
\hline$\Delta E_{\mathrm{mag}}^{(2)}$ & $-\frac{1}{2} \chi_{\mathrm{mag}} \cdot \delta B_{\mathrm{ex}}^{2}$ & - & $-\frac{1}{2} \chi_{\mathrm{mag}} \cdot \delta B_{\mathrm{ex}}^{2}$ \\
& $\chi_{\mathrm{mag}} \simeq-\frac{e^{2} a_{\mathrm{B}}^{2}}{6 m}$ & & $\chi_{\mathrm{mag}} \simeq-2 \frac{\left(I_{\mathrm{p}} A\right)^{2}}{\Delta_{\mathrm{ge}}}$ \\
& $\sim 10^{-29} \mathrm{Am}{ }^{2} / \mathrm{T}$ & & $\sim 10^{-13}-10^{-11} \mathrm{Am} \mathrm{m}^{2} / \mathrm{T}$ \\
\hline
\end{tabular}

therefore have $\Delta E_{\mathrm{el}}^{(1)}=0$, that is, they do not show a linear Stark effect. As indicated by the numbers listed in Table 1, the dipole moments of the qubits are by many orders of magnitudes larger than those of natural atoms. For the charge qubit, $\mu_{\mathrm{el}}=e d$ is determined by the distance $d$ between the island and the gate electrode and can be as large as $10^{4}$ to $10^{5}$ debye $\left(1\right.$ debye $\left.=3.3 \times 10^{-30} \mathrm{Cm}\right)$. In other words, it exceeds the typical values for natural atoms by 4 to 5 orders of magnitude. The same is true for the magnetic dipole moment, which is given by the persistent current $I_{\mathrm{p}}$ circulating around the qubit loop and the loop area $A$. It typically amounts to $10^{4}$ to $10^{5}$ Bohr's magnetons compared to only a single Bohr's magneton for natural atoms. Obviously, the large dipole moments of the qubits are caused by their much larger physical size. Note that $d$ and $A$ are of the order of $\mu \mathrm{m}$ and $\mu \mathrm{m}^{2}$ for the qubits, but only $\AA$ and $\AA^{2}$ for natural atoms. Of course, the large dipole moments of qubits also have a disadvantage. They make the qubits more sensitive to electric and magnetic noise. We also note that large dipole moments are obtained for Rydberg atoms [33, 34, 35], which are highly excited atomic states with much larger atomic diameter. 
So far we have considered only the uncoupled basis states. The finite coupling of $|\downarrow\rangle$ and $|\uparrow\rangle$ results in the superposition states [22]

$$
\begin{aligned}
& |g\rangle=+\cos \frac{\theta}{2} \mathrm{e}^{+i \varphi / 2}|\downarrow\rangle-\sin \frac{\theta}{2} \mathrm{e}^{-i \varphi / 2}|\uparrow\rangle \\
& |e\rangle=+\sin \frac{\theta}{2} \mathrm{e}^{+i \varphi / 2}|\downarrow\rangle+\cos \frac{\theta}{2} \mathrm{e}^{-i \varphi / 2}|\uparrow\rangle
\end{aligned}
$$

with the level spacing

$$
E_{\mathrm{ge}}=\sqrt{\epsilon^{2}+\Delta_{\mathrm{ge}}^{2}} .
$$

Here, $\tan \theta \equiv \Delta_{\text {ge }} / \epsilon$ and $\epsilon \equiv 2 \mu \delta F_{\text {ex }}$ is the energy bias given by

$$
\begin{aligned}
& \epsilon_{\mathrm{el}} \equiv 2 e d \delta E_{\mathrm{ex}}=\frac{(2 e)^{2}}{2 C_{\mathrm{g}}} 2 \delta N_{\mathrm{ex}} \\
& \epsilon_{\mathrm{mag}} \equiv 2 I_{\mathrm{p}} A \delta B_{\mathrm{ex}}=\frac{\Phi_{0}^{2}}{2 L} 2 \delta N_{\mathrm{ex}}
\end{aligned}
$$

for the charge and flux qubit, respectively. The large dipole moments of the qubits allow to considerably tune $E_{\text {ge }}$ by applied control fields. Already fields of the order of $10^{3} \mathrm{~V} / \mathrm{m}$ and $10^{-4} \mathrm{~T}$ are sufficient to cause changes of the order of $\Delta_{\text {ge }}$, whereas for natural atoms the equivalent shifts are usually negligibly small. It is important to note that the expectation values are $\left\langle g\left|\widehat{\sigma}_{z}\right| g\right\rangle=-\cos \theta$ and $\left\langle e\left|\widehat{\sigma}_{z}\right| e\right\rangle=\cos \theta$. Since $\cos \theta=0$ at the degeneracy point, the expectation value of the dipole moments vanish for the superposition states. Therefore, the energy shifts $\Delta E_{\mathrm{el}}^{(1)}$ and $\Delta E_{\mathrm{mag}}^{(1)}$ disappear, making the charge and flux qubit insensitive to low-frequency fluctuations (e.g. $1 / f$ noise) of the electric and magnetic field at this operation point, respectively $[3,4,36]$. Nevertheless, there are significant second-order contributions left, originating from the large polarizabilities of the qubits as discussed in the following.

The second-order terms can be expressed by using the polarizabilities of the natural and artificial atoms. For natural atoms with spherical symmetry, the polarizability tensor is isotropic, giving the quadratic terms $\Delta E_{\mathrm{el}}^{(2)}=-\frac{1}{2} \chi_{\mathrm{el}}$. $\delta E_{\mathrm{ex}}^{2}$ and $\Delta E_{\mathrm{mag}}^{(2)}=-\frac{1}{2} \chi_{\mathrm{mag}} \cdot \delta B_{\mathrm{ex}}^{2}$, where $\chi_{\mathrm{el}}$ and $\chi_{\mathrm{mag}}$ are the electric and magnetic polarizabilities, respectively. Using second-order perturbation theory, the polarizabilities of the qubits can be written as

$$
\chi_{\mathrm{el}, \mathrm{mag}}=-2 \frac{\left\langle g\left|\widehat{\mu}_{\mathrm{el}, \mathrm{mag}}\right| e\right\rangle\left\langle e\left|\widehat{\mu}_{\mathrm{el}, \mathrm{mag}}\right| g\right\rangle}{E_{\mathrm{ge}}}
$$

with $E_{\mathrm{ge}} \equiv E_{\mathrm{e}}-E_{\mathrm{g}}, \widehat{\mu}_{\mathrm{el}}=e d \widehat{\sigma}_{z}$, and $\widehat{\mu}_{\mathrm{mag}}=I_{\mathrm{p}} A \widehat{\sigma}_{z}$. Because $\left\langle g\left|\widehat{\sigma}_{z}\right| e\right\rangle=\left\langle e\left|\widehat{\sigma}_{z}\right| g\right\rangle=$ $\sin \theta$, we obtain the polarizabilities

$$
\begin{aligned}
\chi_{\mathrm{el}} & =-2 \frac{(e d)^{2} \sin ^{2} \theta}{E_{\mathrm{ge}}} \\
\chi_{\mathrm{mag}} & =-2 \frac{\left(I_{\mathrm{p}} A\right)^{2} \sin ^{2} \theta}{E_{\mathrm{ge}}}
\end{aligned}
$$

and the second-order energy shifts

$$
\begin{aligned}
& \Delta E_{\mathrm{el}}^{(2)}=\frac{(e d)^{2} \sin ^{2} \theta}{E_{\mathrm{ge}}} \cdot \delta E_{\mathrm{ex}}^{2} \\
& \Delta E_{\mathrm{mag}}^{(2)}=\frac{\left(I_{\mathrm{p}} A\right)^{2} \sin ^{2} \theta}{E_{\mathrm{ge}}} \cdot \delta B_{\mathrm{ex}}^{2} .
\end{aligned}
$$


Interestingly, the second-order contributions are maximum at the degeneracy point, where the first-order contributions vanish, and decrease moving away from it because of the $\sin ^{2} \theta$ term. Close to the degeneracy point we can use the approximations $\sin \theta \simeq 1$ and $E_{\mathrm{ge}} \simeq \Delta_{\mathrm{ge}}$ and obtain the polarizabilities $\chi_{\mathrm{el}} \simeq-2(e d)^{2} / \Delta_{\mathrm{ge}}$ and $\chi_{\mathrm{mag}} \simeq-2\left(I_{\mathrm{p}} A\right)^{2} / \Delta_{\mathrm{ge}}$, which are listed in Table 1 . Again, due to the much larger size of the qubits the polarizabilities are by many orders of magnitudes larger than those of natural atoms. Since in electrostatics the electric and magnetic energy contributions also can be expressed as $\frac{1}{2} C_{\mathrm{Q}} d^{2} \delta E_{\mathrm{ex}}^{2}$ and $\frac{1}{2}\left(A^{2} / L_{\mathrm{Q}}\right) \delta B_{\mathrm{ex}}^{2}$, the polarizabilities can be associated with a quantum capacitance $C_{\mathrm{Q}}=2 e^{2} / \Delta_{\text {ge }}$ and a quantum inductance $L_{\mathrm{Q}}=\Delta_{\text {ge }} / 2 I_{\mathrm{p}}^{2}$, respectively $[37,38,39,40]$.

\subsection{Coupling Strength and Symmetry Breaking in Circuit QED}

In the previous subsection we have seen that charge and flux qubits show a large tunability of their energy levels due to their large dipole moments and polarizabilities, allowing to tune the level separation over a wide range by static electric and magnetic gate fields. In the following we address the coupling strength with dynamic electric and magnetic fields as well as some fundamental symmetry aspects of superconducting circuit QED. In our analysis we do not only focus on flux qubits discussed in section 2.1. We rather show that the arguments given in the following are quite general and can be straightforwardly applied also to charge qubits.

It is well known [24] that the potential of the three-junction flux qubit can be reduced to the one-dimensional double-well $U(\Phi)$ shown in Fig. 6(a). Here, $\Phi$ is the total magnetic flux in the qubit loop. It originates from the externally applied flux $\Phi_{\mathrm{ex}}$ and the flux due to the current circulating in the loop, having an expectation value $I_{p}\left\langle\widehat{\sigma}_{z}\right\rangle$. We first discuss the situation at the degeneracy point, where the external flux $\Phi_{\text {ex }}$ equals $\Phi^{(n)} \equiv \Phi_{0}(n+1 / 2)$. Here, $n$ is an integer and we only consider $n=0$ in the following. Then, the two wells are symmetrical with respect to $\Phi=\Phi_{0} / 2$ and $I_{p}\left\langle\widehat{\sigma}_{z}\right\rangle$ vanishes. In Fig. 6(a) we also plot the eigenfunctions $|\mathrm{g}\rangle$ and $|\mathrm{e}\rangle$, which, at the degeneracy point, are fully symmetric and antisymmetric superpositions of the two basis states $|\downarrow\rangle$ and $|\uparrow\rangle$ with clock- and counterclockwise circulating persistent currents $\pm I_{\mathrm{p}}$. For a wider region around the degeneracy point, the energy difference $E_{\text {ge }}(\epsilon)$ (cf. eq.(9)) between the two levels is shown in Fig. 6(b). At the degeneracy point, the energy bias $\epsilon=2 I_{\mathrm{p}} A \delta B_{\mathrm{ex}}=2 I_{\mathrm{p}} \delta \Phi_{\mathrm{ex}}$ is zero resulting in $E_{\mathrm{ge}}=\Delta_{\mathrm{ge}}$. The probability to observe $|\downarrow\rangle(|\uparrow\rangle)$ is just 0.5 at the degeneracy point and approaches 1 or 0 (0 or 1) when decreasing or increasing the applied flux. For the charge qubit the situation is completely analogous. At the degeneracy point, the ground state $|\mathrm{g}\rangle$ and the excited state $|\mathrm{e}\rangle$ are fully symmetric and antisymmetric superpositions of the two basis states $|\downarrow\rangle$ and $|\uparrow\rangle$ corresponding to neighboring Cooper pair number states on the superconducting island.

Considering symmetries, we immediately see from Fig. 6(a) that the potential $U$ of the flux qubit is fully symmetric with respect to the variable $\delta \Phi=\Phi-\Phi_{0} / 2$ for an external flux bias $\Phi_{\mathrm{ex}}=\Phi_{0} / 2$. That is, at the degeneracy point we have $U(\delta \Phi)=U(-\delta \Phi)$. We note that $\delta \Phi$ is associated with the phase differences $\gamma_{1}$ and $\gamma_{2}$ across the two larger junctions as $\left(\gamma_{2}-\gamma_{1}\right) / 2=2 \pi\left(\delta \Phi / \Phi_{0}\right)$. In complete analogy, $U(\delta Q)=U(-\delta Q)$ for the charge qubit at an external charge bias of $Q_{\mathrm{ex}}=2 e / 2$. Here, $\delta Q=Q-2 e / 2$ is the quantum variable conjugate to $\delta \Phi$. In both cases the qubit eigenstates $|\mathrm{g}\rangle$ and $|\mathrm{e}\rangle$ have even and odd parity because they are fully symmetric and 

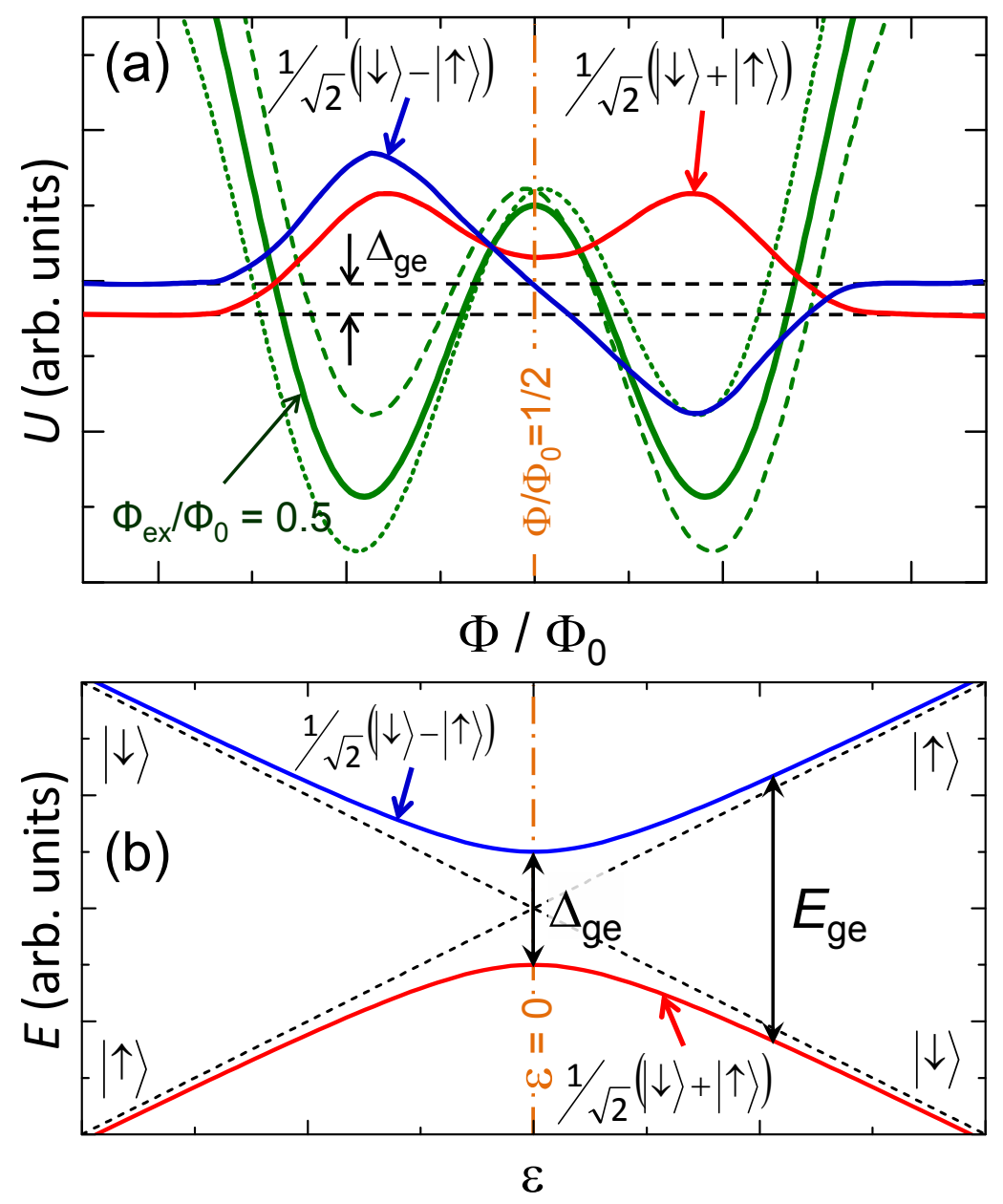

Figure 6. (a) Sketch of the double-well potential $U$ of a flux qubit plotted versus the total flux $\Phi$ in the qubit loop for an externally applied flux $\Phi_{\mathrm{ex}}=\Phi_{0} / 2$ (solid green line). Also shown are the two eigenfunctions of the ground state (symmetric, red) and the first excited state (antisymmetric, blue). The dotted and dashed lines show the qubit potential for an external flux larger and smaller than $\Phi_{0} / 2$. (b) The energy $E$ of the two superposition states $|\mathrm{g}\rangle=1 / \sqrt{2}(|\downarrow\rangle+|\uparrow\rangle)$ and $|\mathrm{e}\rangle=1 / \sqrt{2}(|\downarrow\rangle-|\uparrow\rangle)$ versus the energy bias $\epsilon$. The dashed lines show the energies of the uncoupled basis states.

antisymmetric superpositions of the basis states. Then we can write

$$
\begin{aligned}
& \widehat{\Pi}|\mathrm{g}\rangle=+|\mathrm{g}\rangle \\
& \widehat{\Pi}|\mathrm{e}\rangle=-|\mathrm{e}\rangle,
\end{aligned}
$$

where $\widehat{\Pi}$ is the parity operator. Moving away from the degeneracy point, the potential is tilted to the left or right as indicated by the dotted and dashed lines in Fig. 6(a). Then, symmetry is no longer well defined and $|\mathrm{g}\rangle$ and $|\mathrm{e}\rangle$ are no longer fully symmetric and antisymmetric superpositions of the basis states. In this way, we we can break the symmetry of the flux qubit in a controlled way using an external control parameter, namely the static magnetic flux $\delta \Phi_{\mathrm{ex}}=\Phi_{\mathrm{ex}}-\Phi_{0} / 2$ generated by a magnetic field 
applied via a control gate. For charge qubits, correspondingly the control parameter is the excess charge $\delta Q_{\mathrm{ex}}=2 e \delta N_{\mathrm{ex}}=2 e\left(N_{\mathrm{ex}}-1 / 2\right)$ induced on the island by an electric field applied via a gate capacitor.

The controlled symmetry breaking by static applied gate fields has far-reaching consequences on the selection rules for level transitions. At the degeneracy point, these selection rules can be intuitively understood by the fact that the qubit potential exhibits mirror symmetry, $U(\delta \Phi)=U(-\delta \Phi)$ and $U(\delta Q)=U(-\delta Q)$ for the flux and charge qubit, respectively [cf. Fig. 6(a)]. Hence, the interaction operator of the one-photon driving initiating level transitions between the fully symmetric and antisymmetric states $|g\rangle$ and $|e\rangle$ must be odd with respect to the variable $\delta \Phi_{\mathrm{ex}}$ or $\delta Q_{\mathrm{ex}}[2,41]$. Such odd-parity interaction operators are the dipole operators [cf. eqs.(2), (3), and (4)] for the flux and charge qubit, respectively, because $\sum_{g, e}|i\rangle\left\langle i\left|\widehat{\mu}_{\text {mag,el }}\right| j\right\rangle\langle j| \propto \widehat{\sigma}_{x}$ and, hence, the anticommutator $\left\{\widehat{\Pi}, \widehat{\mu}_{\text {mag,el }}\right\}=0$. In contrast the interaction operator for the two-photon driving is even [2]. Hence, if the interaction operator is a dipole operator, at the degeneracy point only one-photon transitions are allowed, whereas two-photon transitions are strictly forbidden. These selection rules correspond to those for electric dipole transitions in natural atoms [22]. Away from the degeneracy point, the qubit potential no longer exhibits mirror symmetry with respect to $\delta \Phi$ and $\delta Q$ for the flux and charge qubit, respectively, and the superposition states $|g\rangle$ and $|e\rangle$ no longer are purely symmetric and antisymmetric states. Then, the strict selection rules valid at the degeneracy point do no longer apply and one- and two-photon transitions can coexist.

To gain a better insight into this issue one has to explicitly discuss the matrix elements for level transitions in a flux qubit induced by an external microwave driving $\delta B_{\text {ex }}(t)=\delta B_{0} \cos \omega t$ and $\delta E_{\text {ex }}(t)=\delta E_{0} \cos \omega t$ of the flux and charge qubit, respectively. In this context, we first note that for the flux qubit the transitions induced by $\delta B_{\mathrm{ex}}(t)$ are of electric dipole type. This can be understood by recalling that for the flux qubit the magnetic flux $\Phi$ is equivalent to the spatial coordinate, whereas $Q$ is equivalent to the momentum. In contrast, for the charge qubit, the charge $Q$ is equivalent to the spatial coordinate and the flux $\Phi$ to the momentum. With the dipole operator $\widehat{D}(t)=\frac{\Omega}{2} \cos \omega t \widehat{\sigma}_{z}$ and using $\left\langle g\left|\widehat{\sigma}_{z}\right| e\right\rangle=\sin \theta$ we obtain the matrix element for one-photon transitions [22]

$$
\begin{aligned}
\langle g|\widehat{D}(t)| e\rangle & =\frac{\Omega}{2}\left\langle g\left|\widehat{\sigma}_{z}\right| e\right\rangle \cos \omega t \\
& =\frac{\Omega}{2} \sin \theta \cos \omega t,
\end{aligned}
$$

where we define $\Omega=2 I_{\mathrm{p}} A \delta B_{0}$ and $\Omega=2 V_{\mathrm{p}} C d \delta E_{0}=2 e d \delta E_{0}$ for the flux and charge qubit, respectively. Hence, the modulus of the matrix element for one-photon transitions is given by

$$
D^{(1)}=\frac{\Omega}{2} \sin \theta .
$$

For both the flux and charge qubit the transition matrix element is proportional to $\sin \theta=\Delta_{\text {ge }} / E_{\text {ge. }}$. Hence, it is maximum at the degeneracy point $(\sin \theta=1)$ and continuously decreases when moving away from it.

The treatment of the two-photon transitions is more complicated. For $2 \omega \simeq$ $\omega_{\mathrm{ge}}=E_{\mathrm{ge}} / \hbar$ (resonant two-photon driving) the level transitions can be described as a two-step process and a detuning of $E_{\mathrm{ge}} / 2$ [42]. Then, the matrix element for the 

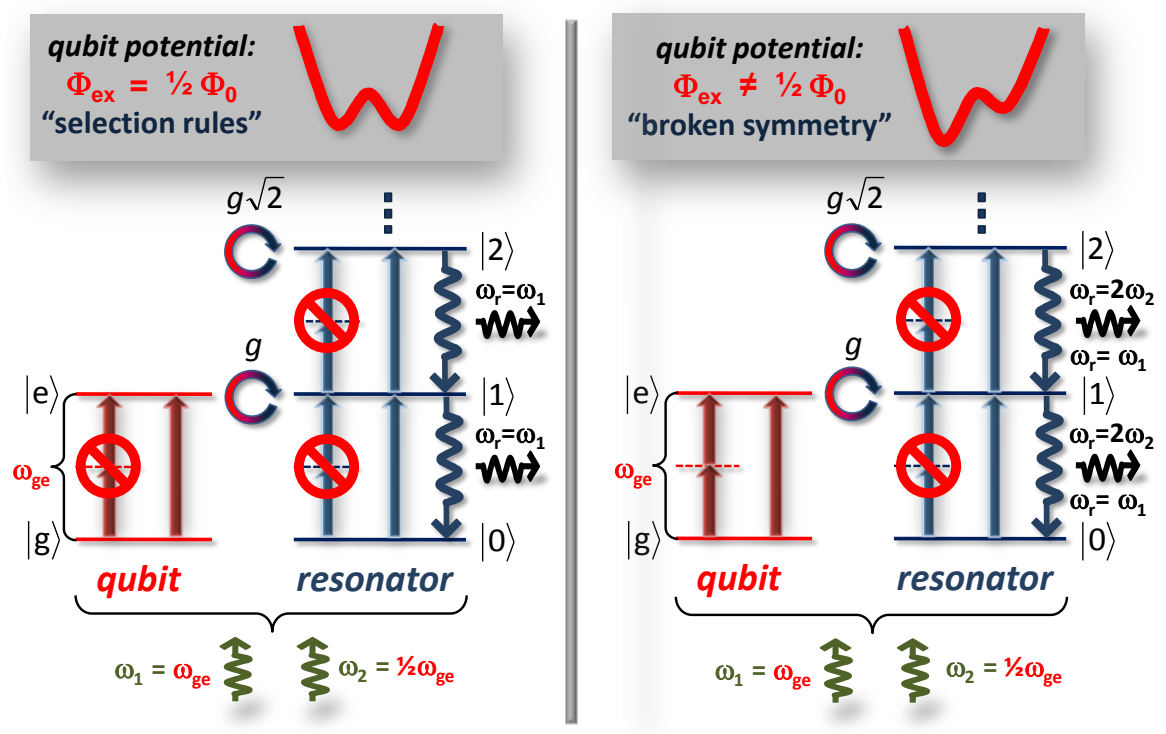

Figure 7. Selection rules for one- and two-photon transitions in a flux qubit having a potential with (left) and without (right) mirror symmetry. In the symmetric situation (left) the two-photon process is forbidden. Since two-photon transitions are also forbidden for the harmonic oscillator, qubit and resonator can be excited only resonantly at frequency $\omega_{1}=\omega_{\mathrm{ge}}=\omega_{\mathrm{r}}$. In contrast, in the case of broken symmetry (right) the qubit can be excited both by one- and two-photon processes. Therefore, also for $\omega_{2}=\omega_{\mathrm{ge}} / 2$ (two-photon process) the excited qubit state $|e\rangle$ can be populated. Due to the qubit-resonator coupling $g / 2 \pi$ a state transfer to the resonator is achieved. The resonator state, in turn, decays by emission of a photon of twice the frequency (frequency up-conversion).

two-photon transition is obtained to

$$
\frac{\langle g|\widehat{D}(t)| e\rangle\langle e|\widehat{D}(t)| e\rangle}{E_{\mathrm{ge}} / 2}=\frac{\Omega^{2}}{4} \frac{\left\langle g\left|\widehat{\sigma}_{z}\right| e\right\rangle\left\langle e\left|\widehat{\sigma}_{z}\right| e\right\rangle}{E_{\mathrm{ge}} / 2} \cos ^{2} \omega t .
$$

With $\left\langle g\left|\widehat{\sigma}_{z}\right| e\right\rangle=\sin \theta,\left\langle e\left|\widehat{\sigma}_{z}\right| e\right\rangle=\cos \theta, \cos ^{2} \omega t=\frac{1}{2}(1+\cos 2 \omega t)$ and $E_{\mathrm{ge}}=\Delta_{\mathrm{ge}} / \sin \theta$ we obtain

$$
\frac{\langle g|\widehat{D}(t)| e\rangle\langle e|\widehat{D}(t)| e\rangle}{E_{\text {ge }} / 2}=\frac{\Omega^{2}}{4 \Delta_{\text {ge }}} \sin ^{2} \theta \cos \theta(1+\cos 2 \omega t) .
$$

From this expression, we identity the modulus of the matrix element for two-photon transitions

$$
D^{(2)}=\frac{\Omega^{2}}{4 \Delta_{\text {ge }}} \sin ^{2} \theta \cos \theta .
$$

The transition matrix elements for both flux and charge qubits are now proportional to $\sin ^{2} \theta \cos \theta=\Delta_{\text {ge }}^{2} \epsilon / E_{\text {ge }}^{3}$. Because $\epsilon=0$ at the degeneracy point, no two-photon transitions are allowed there in agreement with the qualitative discussion above. However, moving away from the degeneracy point the matrix element $D^{(2)}$ becomes finite, making a two-photon driving of the qubit possible. This has been demonstrated in a recent experiment [2], where a flux qubit coupled to an $L C$ resonator has been studied. 
The possibility to deliberately tune the level spacing and the symmetry properties of flux and charge qubits by an external control field can be used to study the effect of symmetry breaking in superconducting circuit QED systems. The key effect of symmetry breaking on selection rules and matrix elements for one- and two-photon transitions in a coupled flux qubit-resonator system is sketched in Fig. 7 for a threejunction flux qubit. The resonator, a harmonic oscillator, can never accept a twophoton driving. Furthermore, for an external flux bias $\Phi_{\mathrm{ex}}=\Phi_{0} / 2$ the qubit potential has perfect mirror symmetry (left) and the selection rules discussed above hold. Then, qubit and resonator can be excited only resonantly at frequency $\omega_{1}=\omega_{\text {ge }}$. By changing the flux bias away form $\Phi_{0} / 2$, the mirror symmetry of the potential is broken (right). In this case, also for two-photon driving $\left(\omega_{2}=\omega_{\mathrm{ge}} / 2\right)$ the excited qubit state $|e\rangle$ can be populated. The strong qubit-resonator coupling $g / 2 \pi$ results in a state transfer to the resonator, which subsequently decays by emission of a photon of twice the frequency. This frequency up-conversion mechanism for microwave photons in a coupled qubit-resonator system has been studied in a recent experiment [2]. We note that the occurrence of this process is a direct consequence of the underlying symmetry properties of the system, which can be chosen on purpose by an external control parameter (magnetic flux in our case). The symmetry properties of the qubitresonator system have an interesting consequence. Depending on the choice of $\Phi_{\text {ex }}$ and the microwave frequency $\omega / 2 \pi$, one can choose to drive either only the qubit, only the resonator, or both the qubit and the resonator. This selective driving once more demonstrates the benefits of the tunability of solid-state circuit QED systems. Besides its role in fundamental research, the controlled symmetry breaking in artificial quantum systems has good prospects of use in numerous applications. Particular examples are parametric up-conversion, the generation of single microwave photons on demand [10, 12, 43], and squeezing of quantum states [44].

\section{Conclusions}

We have fabricated superconducting three-junction flux qubits using electron beam lithography and two-angle shadow evaporation. The qubits are based on $\mathrm{Al} / \mathrm{AlO}_{x} / \mathrm{Al}$ Josephson junctions with areas down to $0.02 \mu \mathrm{m}^{2}$ and high current densities above $1 \mathrm{kA} / \mathrm{cm}^{2}$. They show a level splitting above $4 \mathrm{GHz}$ at $50 \mathrm{mK}$. We also fabricated readout dc SQUIDs and additional on-chip circuit elements such as shunting capacitors for the readout SQUID, transmission lines for the application of microwave signals to the qubits, and coplanar waveguide resonators. The latter are based on $\mathrm{Nb}$ films and have resonance frequencies ranging between about 1 and $10 \mathrm{GHz}$. They show internal quality factors above $10^{5}$ at $1.5 \mathrm{~K}$.

We also studied the tunability of superconducting charge and flux qubits by external control fields. We derived the effective dipole moments of the qubits and show that they are by several orders of magnitude larger than those of natural atoms because of the much larger geometrical size of the artificial solid-state atoms (qubits). Furthermore, we derived the first- and second-order energy shifts of the qubit levels due to control fields as well as expressions for the matrix elements describing the level transitions at and away from the degeneracy point. We finally addressed some fundamental symmetry aspects of superconducting qubits. We argue that at the degeneracy point the qubit potential has mirror symmetry resulting in selection rules for level transitions equivalent to those in natural atoms. However, the symmetry of the qubit potential can be easily broken by external control fields. Then, the strict 
selection rules are no longer valid. In particular, two-photon transitions are allowed resulting in an interesting up-conversion dynamics in a coupled qubit-resonator system, which we could observe in a recent experiment.

\section{Acknowledgments}

This work is supported by the German Science Foundation via SFB 631 and the German Excellence Initiative via the Nanosystems Initiative Munich (NIM).

\section{References}

[1] Nielsen MA and Chuang IL 2000 Quantum Computation and Quantum Information (Cambridge Univ. Press)

[2] Deppe F, Mariantoni M, Menzel EP, Marx A, Saito S, Kakuyanagi K, Tanaka H, Meno T, Semba K, Takayanagi H, Solano E and Gross R 2008 Nature Physics 4686

[3] Kakuyanagi K, Meno T, Saito S, Nakano H, Semba K, Takayanagi H, Deppe F and Shnirman A 2000 Phys. Rev. Lett. 98047004

[4] Deppe F, Mariantoni M, Menzel EP, Saito S, Kakuyanagi K, Tanaka H, Meno T, Semba K, Takayanagi H and Gross R 2007 Phys. Rev. B 76214503

[5] Clarke J and Wilhelm FK 2008 Nature 4531031

[6] Schoelkopf RJ and Girvin SM 2008 Nature 451664

[7] Wallraff A. et al. 2004 Nature 431162

[8] Chiorescu I. et al. 2004 Nature 431, 159

[9] Blais A, Huang RS, Wallraff A, Girvin SM and Schoelkopf RJ 2004 Phys. Rev. A 69062320

[10] Mariantoni M et al. 2005 eprint arXiv:cond-mat/0509737 (unpublished).

[11] Johansson J, Saito S, Meno T, Nakano H, Ueda M, Semba K and Takayanagi H 2006 Phys. Rev. Lett. 96127006

[12] Houck AA et al. 2007 Nature 449328

[13] Sillanpää MA, Park JI and Simmonds RW 2007 Nature 449438

[14] Majer J et al. 2007 Nature 449443

[15] Astafiev O et al. 2008 Nature 449588

[16] Hofheinz $\mathrm{M}$ et al. 2008 Nature $\mathbf{4 5 4} 310$

[17] Fink JM et al. 2008 Nature $\mathbf{4 5 4} 315$

[18] Mariantoni M, Deppe F, Marx A, Gross R, Wilhelm FK and Solano E 2008 Phys. Rev. B 78 104508

[19] Mabuchi H and Doherty AC 2002 Science 2981372

[20] Haroche S and Raimond JM 2006 Exploring the Quantum: Atoms, Cavities, and Photons (Oxford Univ. Press)

[21] Walther H, Varcoe BTH, Englert BG and Becker T 2006 Rep. Prog. Phys. 691325

[22] Cohen-Tannoudji C, Diu B and Laloë F 1977 Quantum Mechanics (WileyInterscience, New York)

[23] Mooij JE, Orlando TP, Levitov LS, Tian L, van der Wal CH and Lloyd S 1999 Science 285, 1036

[24] Orlando TP, Mooij JE, Tian L, van der Wal CH, Levitov LS, Lloyd S and Mazo JJ 1999 Phys. Rev. B 6015398

[25] van der Wal CH, ter Haar ACJ, Wilhelm FK, Schouten RN, Harmans CJPM, Orlando TP, Lloyd S and Mooij JE 2000 Science 290773

[26] Dolan GJ 1977 Appl. Phys. Lett. 31337

[27] Ambegaokar V and Baratoff A 1963 Phys. Rev. Lett. 10486

[28] Deppe F, Saito S, Tanaka H and Takayanagi H 2004 J. Appl. Phys. 952607

[29] Wallraff A et al. 2007 Phys. Rev. Lett. 99050501

[30] Frunzio L, Wallraff A, Schuster D, Majer J, Schoelkopf R 2005 IEEE Trans. Appl. Supercond. 15, 860

[31] Pozar DM Microwave Engineering 2005 (John Wiley \& Sons)

[32] Girfoni M, Hänggi P 1998 Phys. Rep. 304229

[33] Meschede D, Walther H. and Muller G 1985 Phys. Rev. Lett. 54551

[34] Rempe G, Walther H. and Klein N 1985 Phys. Rev. Lett. 58353.

[35] Raimond JM, Brune M. and Haroche S 2001 Rev. Mod Phys. 73565 
[36] Yoshihara F, Harrabi K, Niskanen AO, Nakamura Y and Tsai JS 2006 Phys. Rev. Lett. 97 167001

[37] Averin DV and Bruder C 2003 Phys. Rev. Lett. 91057003

[38] Sillanpää MA, Lehtinen T, Paila A, Makhlin Yu, Roschier L and Hakonen PJ 2005 Phys. Rev. Lett. 95206806

[39] Duty T, Johansson G, Bladh K, Gunnarsson D, Wilson C and Delsing P 2005 Phys. Rev. Lett. 95206807

[40] Könemann J, Zangerle H, Mackrodt B, Dolata R and Zorin AB 2007, Phys. Rev. B 76134507

[41] Liu Y-X, You JQ, Wei LF, Sun CP and Nori F 2005 Phys. Rev. Lett. 95087001

[42] Brune M, Raimond JM, Haroche S 1987 Phys. Rev. A 35154

[43] Liu Y-X, Wei LF and Nori F. 2004 Europhys. Lett. 67, 941

[44] Moon K and Girvin SM 2005 Phys. Rev. Lett. 95140504 